\title{
Progress in the Application of Single-cell Sequencing Technology in Cartilage Tissue Diseases
}

\author{
Li Zhencong ${ }^{1}$, Xu Yude ${ }^{1}$, Wu Hao ${ }^{2}$, Yao Yicun ${ }^{1}$, Ye Dongping ${ }^{1}$, Miao Haixiong ${ }^{1}$, Liang Weiguo ${ }^{1, ~ * ~}$ \\ ${ }^{1}$ Department of Orthopedics, Guangzhou Red Cross Hospital, Medical College, Jinan University, Guangzhou, China \\ ${ }^{2}$ School of Clinical Medicine, Guizhou Medical University, Guiyang, China
}

Email address:

lizhencong718@163.com (Li Zhencong), liangweiguo1011@126.com (Liang Weiguo)

${ }^{*}$ Corresponding author

\section{To cite this article:}

Li Zhencong, Xu Yude, Wu Hao, Yao Yicun, Ye Dongping, Miao Haixiong, Liang Weiguo. Progress in the Application of Single-cell Sequencing Technology in Cartilage Tissue Diseases. International Journal of Clinical and Experimental Medical Sciences. Vol. 7, No. 4, 2021, pp. 120-126. doi: 10.11648/j.ijcems.20210704.19

Received: July 30, 2021; Accepted: August 10, 2021; Published: August 26, 2021

\begin{abstract}
Background: In the study of the pathogenesis of cartilage tissue (articular cartilage, meniscus, intervertebral disc, etc.) diseases, the most common research object is to explore some possible pathogenesis and pathogenesis in the direction of the whole pathological changes of these overall tissues. Therefore, there are many limitations and inaccuracies in the previous research methods. Single-cell sequencing refers to the technology of sequencing the genome and transcriptome epigenome at the single cell level, which can obtain the heterogeneity data between different cells from the sample tissue and sequence the precious very trace sample, so as to reflect the law and essence of life at a deeper level. Objective: Single cell sequencing technology can be used to explore the pathogenesis of cartilage tissue diseases, understand the relationship between a cell type and diseases and provide new ideas for treatment and diagnosis. Methods: The first author used the Chinese search terms "single-cell sequencing, cartilage, intervertebral disc, meniscus" and the English search terms "single-cell sequencing; cartilage; intervertebral disc; meniscus, osteoarthritis", relevant articles included between January 2010 and April 2021 were searched in Embase, PubMed, Wanfang database, and China National Knowledge Infrastructure (CNKI) database. The retrieved relevant literatures were screened and collated, and the included relevant literatures were reviewed in detail. Results \& Conclusion: From the emergence of single-cell sequencing technology for the first time more than ten years ago, single-cell sequencing technology has been rapidly developed, and occupies an important position in the whole history of life science and technology development. Single-cell sequencing has been widely used in the study of tumor heterogeneity, immune microenvironment, neuroscience, embryonic development, and cell differentiation. However, the application in cartilage tissue diseases, is still in the primary stage of exploratory research. Single-cell sequencing technology can better understand the pathogenesis of cartilage tissue diseases at the cellular level, providing the possibility for pathological staging and targeted therapy at the molecular level of the disease. This is helpful for early diagnosis and early intervention of cartilage tissue diseases, reducing the pain caused by advanced cartilage tissue diseases and the economic burden of the whole society, so it also has a broad prospect in cartilage tissue diseases.
\end{abstract}

Keywords: Single Cell Sequencing, Cartilage Tissue, Disc, Meniscus, Articular Cartilage, Osteoarthritis

\section{Introduction}

Single cell sequencing refers to a new technology for high-throughput sequencing analysis of epigenome, transcriptome and genome at the level of single cell. It can compensate for the limitations of previous traditional high-throughput sequencing, reveal the interaction relationship between different cells between tissues, as well as the gene structure and gene expression status of single cells, and reflect the heterogeneity between cells. Compared with the traditional sequencing technology that only reflects the specificity of a certain tissue or cell population, single-cell sequencing technology can reveal the differences in the expression of genetic material and protein at the level of a single cell, so as to better understand the function of a single cell in a specific environment and specific tissue. At present, single-cell 
sequencing has been widely used in various fields: such as tumor heterogeneity [1], immune microenvironment [2], neuroscience [3], embryonic development [4], and cell differentiation [5]. Moreover, with the continuous development of single-cell sequencing technology and the continuous improvement and innovation of sequencing methods, it will be applied to the field of more biological disciplines in the future. It can be used to explore its pathogenesis, understand the relationship between a cell type and the disease, and provide new ideas for treatment and diagnosis in cartilage tissue diseases, which are briefly summarized in this paper.

\section{Materials and Methods}

\subsection{Search Strategy}

Source of data The first author searched the Chinese search terms "single-cell sequencing; cartilage; intervertebral disc; meniscus; osteoarthritis" and the English search terms "single-cell sequencing; cartilage; meniscus; intervertebral disc; osteoarthritis", relevant articles included between January 2011 and March 2021 were searched in Embase,
PubMed, Wanfang database, and China National Knowledge Infrastructure (CNKI) database. According to the selection criteria, the relevant literatures were screened and sorted out, and the included relevant literatures were reviewed in detail.

\subsection{Selection Criteria}

Inclusion criteria: 1), Selected the relevant literature data of articular cartilage, meniscus and intervertebral disc in single-cell sequencing samples; 2), Chinese or English literatures must have the characteristics of good quality, high relevance and strong timeliness; 3), Relevant research content and data analysis must be authentic.

Exclusion criteria: 1), literatures with poor quality, low relevance and repeatability; 2), unreliable literature content and data analysis.

\subsection{Quality Assessment and Data Extraction}

A total of 271 relevant articles were retrieved, including 31 Chinese articles and 240 English articles. 271 articles were screened according to the selection criteria, and a total of 52 Chinese and English articles that met the inclusion criteria were included, as shown in Figure 1.

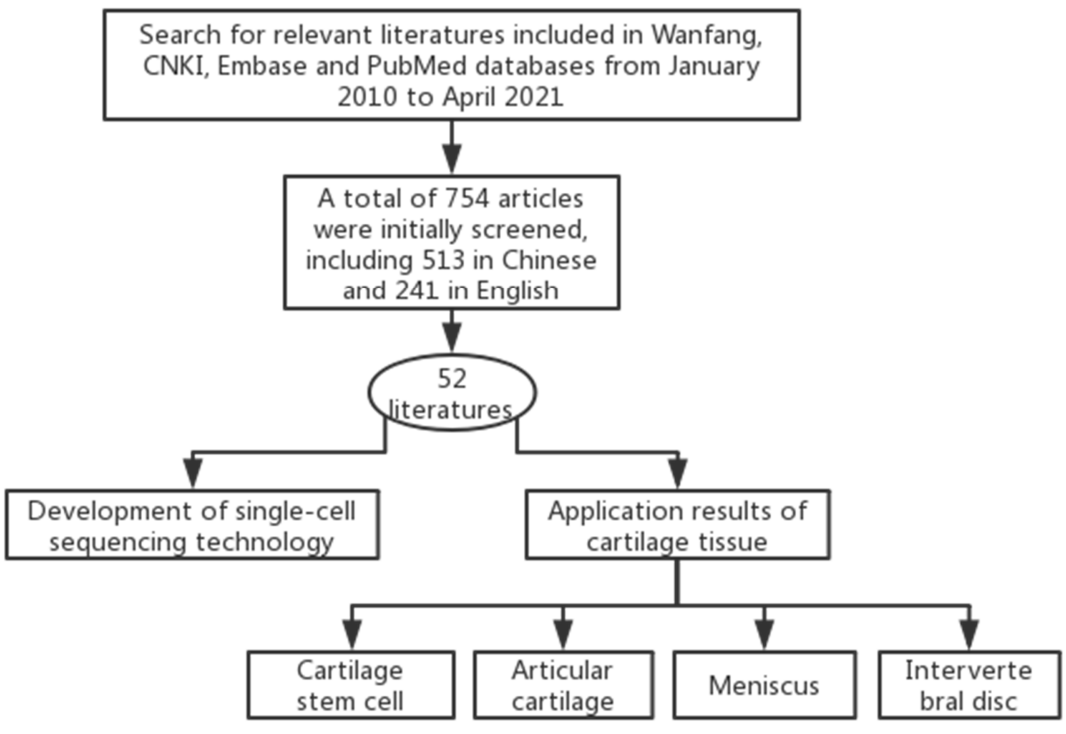

Figure 1. Literature Screening flow chart.

\section{Results}

\subsection{Single-cell Gene Transcriptome Sequencing Technology}

\subsubsection{Sequencing Overview of Single-cell Gene Transcriptomes}

Transcriptome refers to all RNA transcribed from a cell at a specific stage of a cell. These include mRNAs and non-coding RNAs [6-7]. Transcriptomics is to study the expression of intracellular genes from the level of IIIIA, and to study the situation of gene transcription and the law of gene transcription regulation in individual cells at the overall level.
Unlike the genome, transcriptomics studies encompass the time and space constraints of individual cells. The gene expression of the same cell is not exactly the same in different growth periods and growth environments. In recent years, the emerging single-cell sequencing technology can amplify and sequence the whole transcriptome at the single-cell level, and its technical principle is to amplify the trace whole transcriptome RNA of a single cell isolated from the tissue followed by high-throughput sequencing. Using this technique is able to reveal gene expression status and gene structure information at the global level within individual cells and accurately reflect the heterogeneity between cells. Insights into the interrelationships between cellular genotypes and phenotypes. Single-cell genome technology has been 
applied to multiple subject areas, such as plant cells, animal cells (including human cells) and microorganisms. Single-cell genome sequencing mainly includes four steps: single-cell isolation $\rightarrow$ whole-genome amplification $\rightarrow$ high-throughput sequencing $\rightarrow$ data analysis. Among them, single cell isolation and whole genome amplification play a key role in the accuracy of the final results [8].

\subsubsection{Single Cell Isolation}

The commonly used single cell separation methods mainly include: continuous dilution method, micromanipulation method and laser capture microdissection method, magnetic bead-activated cell sorting method and fluorescence-activated cell sorting method, and microfluidic control method. Microfluidic technology, which has been proposed in recent years, achieves the separation of single cells by constructing a microflow channel with a diameter of $10 \sim 100$ microns to artificially control fluid flow [9-10]. It has the advantages of accurate control, less samples and reagents required, high resolution circuit and high sensitivity. The microfluidic system team led by $\mathrm{Ma} \mathrm{Bo}$, a researcher at the Chinese Academy of Sciences, invented the FOCOT technology with only one cell (one-cell-one-tube) per tube by designing a simple and efficient single-cell sorting and sequencing docking device, which can be accurately, high-speed, and low-cost separated, obtained, and distributed with a single microbial cell, so as to directly dock with single-cell sequencing [11]. At present, many commercial microfluidic platforms have been widely used, such as Single Cell3 Solution introduced by $10 \mathrm{XGenomics}$ that can sort tens of thousands of cells at a time. This sorting platform provides convenience for single-cell genome and transcriptome sequencing, reduces the research cost, and has a broad application prospect [12].

\subsubsection{Single-cell Nucleic Acid Sequence Amplification}

The nucleotide sequence of DNA or RNA in a single cell isolated from a sample is only picogram and cannot be applied to the actual sequencing process, so amplification is required: whole genome amplification (WGA)/whole transcriptome amplification (WTA). Traditional whole genome amplification methods are: PCR-based WGA method, multiple strand displacement amplification (MDA), and multiple annealing circular amplification technique (MALBAC). There are many methods of PCR-based WGA, but PEP-PCR, DOP-PCR and DDOP-PCR are common. Among them, the high amplification bias of DOP-PCR (only certain regions of the genome are preferentially amplified, so it is suitable for large-scale sequencing) leads to relatively low coverage of the genome (about 10\%), making DOPPCR useful in single-cell copy number assessment, but not suitable for single-nucleotide variation (SNV) detection [13]. MDA is a non-PCR amplification technique that does not require thermal cycling, and MDA is most suitable for mutation detection, but it is insufficient for copy number analysis due to moderate amplification bias and uneven genome coverage [14]. MALBAC is a new single-cell whole genome amplification technology published in Science by the
Xiaoliang Xie team at Harvard University in 2012 and is a linear amplification modality. Compared with MDA, MALBAC has a higher efficiency in detecting copy number variations and single nucleotide polymorphisms [15], with the disadvantage of a false positive rate. Recently, Academician Xie Xiaoliang proposed a novel method for linear amplification of single-cell genomes in Science, LIANTI, which has greatly improved all indicators such as amplified gene coverage and fidelity compared with MALBAC, DOP-PCR, and MDA techniques [16]. Commonly used whole transcriptome amplification methods are: Smart-seq/Smart-seq2, Quartz-seq, MATQ-seq, CEL-seq/CEL-seq2, and STRT.

\subsubsection{Single-cell Sequencing Technology}

Single-cell RNA sequencing technology is the discovery of new transcripts by calculating the expression of different RNAs through statistically relevant read segments. There are two main categories: full-length sequencing and tag-based. Full-length sequencing is mainly used to detect and identify the variation and cleavage process of a single base in an allele, while tag-based sequencing is a molecular marker combined with its specificity to target more samples and increase the quality and throughput at the gene level [17]. The Tang's method, proposed in 2009 [18], performs single-cell RNA amplification simultaneously with sequencing technology. On this basis, Sasagawa $\mathrm{Y}$ et al optimized it to reduce amplification by-products and simplify the experimental process, and proposed the method of Quartz-Seq [19]. CELseq with IVT instead of PCR, SMART-based STRT method (single-cell tagged reverse transcription), Smart-Seq and Smart-Seq2 that enable the full-length of transcripts to be sequenced, and the recently Austrian researchers proposed a single-cell sequencing method called CROP-seq in Nature Methods, enable the large-scale completion of unprecedented analysis of high-throughput gene regulation [20], as well as the DroNc-Seq single-cell expression profiling technology proposed by the Zhang Feng team of the Broad Institute in Nature Methods in the same year [21].

\subsection{Application of Single Cell Sequencing in Cartilage Tissue Diseases}

\subsubsection{Application of Single-cell Sequencing in Chondrocyte Stem Cell Induction Culture}

In the past, the advent of human induced pluripotent stem cells (hiPSCs) has served as a major breakthrough in in vitro disease modeling for cartilage regeneration therapy and osteoarthritis (OA) drug discovery [22]. However, developing protocols for continuously differentiating hiPSCs into chondrocytes remains challenging. Early studies reported that hiPSCs can generate chondrocytes by expansion of mesodermal cell monolayers after embryoid body formation and culture in three-dimensional cell pellets in chondrogenic induction medium [23, 24]. Therefore, recent researchers are all trying to use serum-free and chemically defined media [25 - 27]. By coupling induction and inhibition 
signals required for specification of the developing mesoderm of the embryo [28], Chia-Lung $\mathrm{Wu}$ et al. established a stepwise chondrogenic differentiation protocol for hiPSC by using multiple hiPSC lines in several laboratories [29].

Chia-Lung Wu et al applied bulk RNA sequencing (bulk RNA-seq) and single-cell RNA sequencing (scRNA-seq) to map the kinetics of gene expression during mesodermal and chondrogenic differentiation of hiPSCs [30]. By using single-cell transcriptomics, the team confirmed mesodermal and chondrogenic differentiation of hiPSCs, in addition to identifying GRNs and key hub genes that regulate heterogeneous off-target cell generation. At the same time, it was demonstrated that the homogeneity of chondrogenesis in hiPSC could be significantly improved by inhibiting the molecular targets WNTs and MITF. This study developed and validated an enhanced chondrogenic differentiation protocol for hiPSC.

At the same time, in order to determine the phenotype of hiPSC-derived cartilage, the team made projections in PCA for the large amount of RNA-seq data from this study and publicly available sequencing datasets of primary chondrocytes from various cartilage tissues and chondrocytes from human mesenchymal stem cells (hMSCs) [31]. HiPSC-derived chondrocytes were therefore found to exhibit a phenotype similar to embryonic limb bud chondrocytes.

\subsubsection{Application of Single-cell Sequencing in Meniscus}

Hao Sun et al. [32] found seven cell clusters, including five previously discovered cell clusters and two new cell clusters, by single-cell high-throughput scRNA sequencing of healthy individuals and degenerated menisci, specifically identifying the following cells: (1) endothelial cells (EC, expressing CD93 and CDH5), [33] (2) chondroprogenitor cells (CPC, expressing CDK1 and BIRC5), [34] (3) regulatory chondrocytes (RegC, expressing BMP2 and FOSL1), [35] (4) fibrochondrocytes (FC, expressing COL1A1, COL3A1, and COL6A1), [34, 36] (5) Prehypertrophic chondrocytes (PreHTC, expressing MMP1 and TNFAIP6), [37-38]. fibrochondrocyte progenitor cells (FCP, which express both the fibrochondrocyte genes COL1A1 and COL3A1 as well as the intercellular stem cell marker genes MCAM and MYLK) [39] and (7) fibrochondrocyte proliferation (ProFC, which express both the fibrochondrocyte genes COL1A1 and the growth factors FGF7 and CTGF) [40].

Inverse time analysis revealed the presence of endothelial cells and fibrochondrocyte progenitors at the origin of the pseudospatial trajectory. Melanoma cell adhesion molecule (McaM)/cD146) was highly expressed in two clusters. CD146 + meniscal cells differentiated into osteoblasts and adipocytes and formed colonies. We identified changes in the proportion of degenerating meniscal cell clusters and found a degenerative meniscus-specific cluster with progenitor cell characteristics. The reconstitution of four progenitor clusters suggests that $\mathrm{FcP}$ differentiation into DegP is an aberrant process. In healthy human meniscal cells, interleukin 1 stimulation increased cD318 + cells, whereas TGF1 attenuated the increase in $\mathrm{cD} 318+$ cells in degenerated meniscal cells.

The researchers identified the cells and their gene signatures of healthy and degenerated meniscus cells to determine their differentiation relationships and characterize the diversity within specific cell types. Colony formation, multi-differentiation assays, and mouse meniscal injury models were used to identify meniscal progenitor cells. The role of degenerated meniscus progenitor cell (DegP) cell mass in the process of meniscus degeneration was studied by computational analysis and experimental validation.

Hao Sun et al suggested that CD146 + meniscal cells have potential in meniscal tissue engineering and DegP could be a possible therapeutic target for meniscal degeneration. The identification of meniscal progenitor cells has provided new insights into cell-based meniscal tissue engineering, demonstrating novel mechanisms of meniscal degeneration, which could help to develop a new therapeutic strategy.

\subsubsection{Application of Single-cell Sequencing in Human Arthritis}

Osteoarthritis (OA) is the most common form of arthritis involving the joints, including the knee, hip, finger interphalangeal, and spinal facet joints. And it usually occurs after middle age, causing joint pain and limited mobility [41]. $\mathrm{OA}$ is a major cause of lower limb disability in the elderly. An estimated 2.4 billion people worldwide suffer from osteoarthritis [42]. Cartilage is the main lesion site and has been the focus of OA research. However, the causes related to the gene molecular level and cellular level of cartilage diseases are still not clear.

Xiaoyu Li et al explored and compared the genes and their expression patterns that play an important role in the pathogenesis of OA by RNA-seq technique using 18 normal knee cartilage tissues and 20 OA affected tissues [43].

Differential gene expression analysis: Based on PCA results, there were some differences between normal and OA samples: the team obtained 1375 up-regulated DEGs and 1026 down-regulated DEGs. According to the adjusted p-value ranking, the top five DEGs up- or down-regulated were CFI, SULF1, SPOCK1, FUT4, GRIA2 and DDIT3, MAFF, CISH, BCOR, ADM.

Weighted gene co-expression network analysis (WGCNA): When a gene is positively correlated with $\mathrm{OA}$, it is also strongly positively correlated with age and weakly negatively correlated with males. The modules with the strongest positive and negative correlations with OA contained 1240 and 731 genes, respectively. The hub genes of the modules with the strongest positive and negative correlations with $\mathrm{OA}$ were SLC35B4 and MAFF, respectively. The module genes with the strongest positive correlation with OA were mainly enriched in extracellular matrix-associated pathways, and the module enrichment results with the strongest negative correlation with OA again showed FoxO and HIF-1 signaling pathways.

Quasi-temporal analysis: The study obtained 3139 genes 
that changed as a function of pseudo-time. In the distribution map sorted by time-mimicking cells, CCL3, CXCL8, and IL1B were mainly upregulated at the beginning of the sham time, COL1A1, COL1A2, and PRG4 were upregulated near the end, and FGF1, KRT17, and NGF were upregulated in the middle.

Xiaoyu Li et al intersected genes suggested by the analysis of bulk RNA sequencing (bulk RNA-seq) datasets with genes suggested by the pseudo-temporal analysis of single-cell RNA sequencing (scRNA-seq) datasets. According to the results of bulk RNA-seq and scRNA-seq analysis, 271 shared genes were found, of which 183 were upregulated in osteoarthritic cartilage, while in normal cartilage, 88 were downregulated. Of the 271 shared genes, the team identified 14 transcription factors and 8 long chain non-coding NRAs whose expression patterns were highly consistent with previous studies [44-48].

The study by Xiaoyu Li's team may provide some inspiration for the understanding and treatment of OA.

Quanbo Ji [49] et al performed unbiased whole transcriptome ScRna-seq analysis, computational analysis, and histological analysis of 1464 chondrocytes from 10 patients with OA (osteoarthritis) who underwent knee arthroplasty. As a result, seven molecularly defined chondrocyte populations were identified in human cartilage, including three new phenotypes with different functions: ProCs (proliferating chondrocytes), preHTCs (prehypertrophic chondrocytes), FCs (fibrochondrocytes), HTCs (hypertrophic chondrocytes), EC (effector chondrocytes), RegCs (regulatory chondrocytes), and HomCs (homeostatic chondrocytes).

The research team used the Monocle method based on single-cell data. The axis of the pseudospatial trajectory derived from Monocle was found to match the cell type well, and the cell arrangement in the pseudospatial trajectory corresponded to the spatial relationship of the cell, suggesting that the pseudospatial trajectory demonstrates the similarity of the cell in space. ECs are distributed at the beginning of the trajectory, and RegCs and HomCs are present along the trajectory. ProCs occupy the front of preHTCs and HTCs, and FCs are mainly distributed at the ends. The OA distribution of the cell populations was also analyzed: ECs and RegCs were mainly early OA chondrocytes, whereas preHTCs, HTCs, and FCs were mainly late OA chondrocytes. Moreover, pairwise correlation analysis revealed close relationships between ECs and ProCs, RegCs and ProCs, preHTCs and FCs, and HomCs and HTCs. The team performed EC and $\mathrm{RegC}$ identification, determined the relationship between ProCs, preHTCs, and HTCs, revealed the progress of the two within HTCs, identified the potential functions of FCs and CPCs, and characterized HomCs.

These data suggest that single-cell transcriptional programs can provide potential clues for the treatment of OA.

\subsubsection{Application of Single-cell Sequencing in Non-degenerated Intervertebral Discs}

Lorenzo M. Fernandes et al. [50] suggested that understanding the transcriptomic characteristics of AF and NP compartments in healthy, non-denatured human discs can help understand the basic biology of human discs, help inform the development of novel therapies, and improve the efficacy of stem cell and tissue engineering based regenerative therapies for IDD. The team used NP and AF cells isolated from non-degenerated human discs using an unbiased single-cell ScRNA-seq approach with the aim of identifying unique gene expression profiles that distinguish each compartment.

The team analyzed the expression of 12323 human genes from about $725 \mathrm{AF}$ and $1010 \mathrm{NP}$ single cells. The T-distributed random neighborhood embedding (T-SNE) method was then used to perform unsupervised analysis of cell clusters based on transcriptome profiles to define gene expression heterogeneity in NP and AF cells at the single-cell level. T-SNE analysis revealed separation of NP and AF cells into two distinct clusters, which suggests the presence of two transcriptionally discrete cell populations. Finally, it is concluded that NP and AF express cell type-specific markers and separate them into different cell populations. ScRNA-seq data reveal distinct transcriptional differences between the two compartments of the human disc that can be expected due to the different developmental origins of these two cell types [51, 52].

This analysis revealed that FOXM1 is the main transcription factor regulating the AF network cluster, while KDM4E is the main transcription factor regulating the NP network cluster. As well as differentially expressed genes between AF and NP and so on.

In this study, Lorenzo M. Fernandes et al bridged some of these gaps in knowledge by identifying AF and NP-enriched genes in cells isolated from non-degenerating, healthy human lumbar discs. In addition, the team mapped the transcriptional landscape of ECM-related genes in both compartments of the human disc and identified a number of transcription factors that may regulate the transcriptional profile of AF and NP cells.

\section{Discussion}

From the first appearance of single-cell sequencing technology more than ten years ago, to 2013, single-cell sequencing technology was first rated as the annual technology by Nature Methods and the top six areas of greatest annual attention by Science, to 2015, single-cell sequencing technology was once again on the cover of science translational medicine, single-cell sequencing technology has been rapidly developed, and occupies an important position in the whole history of life science and technology development. Single-cell sequencing has been widely used in the study of tumor heterogeneity, immune microenvironment, neuroscience, embryonic development, and cell differentiation. However, the application in motor system diseases, especially in cartilage tissue diseases, is still in the primary stage of exploratory research.

Compared with previous reviews, such as "Application of 
single-cell sequencing technology in osteoarticular diseases" published in the Orthopedic Journal of China by Zhang Yu et al.[53], this paper summarizes the updated research results. More innovative than before.

\section{Conclusions}

Single-cell sequencing technology can better understand the pathogenesis of cartilage tissue diseases at the cellular level, providing the possibility for pathological staging and targeted therapy at the molecular level of the disease, such as sequencing the cells in different stages of cartilage tissue degenerative diseases, understanding the distribution of cells in this cell group of chondrocytes, understanding the possible role of different cells in different stages of the disease, so as to find the information of a certain correlation between cells and the disease. This is helpful for early diagnosis and early intervention of cartilage tissue diseases, reducing the pain caused by advanced cartilage tissue diseases and the economic burden of the whole society, so it also has a broad prospect in cartilage tissue diseases.

\section{References}

[1] Kim C, Gao R, Sei E, et al. Chemoresistance Evolution in Triple-Negative Breast Cancer Delineated by Single-Cell Sequencing. Cell 2018, 173 (4): 879-893.

[2] Zheng C, Zheng L, Yoo JK, et al. Landscape of Infiltrating T Cells in Liver Cancer Revealed by Single-Cell Sequencing. Cell 2017, 169 (7): 1342-1356.

[3] Li CL, Li KC, Wu D, et al. Somatosensory neuron types identified by high-coverage single-cell RNA-sequencing and functional heterogeneity. Cell Res 2015, 26 (1): 83-102.

[4] Li L, Dong J, Yan L, et al. Single-Cell RNA-Seq Analysis Maps Development of Human Germline Cells and Gonadal Niche Interactions. Cell Stem Cell 2017, 20 (6): 858-873.

[5] Bach K, Pensa S, Grzelak M, et al. Differentiation dynamics of mammary epithelial cells revealed by single-cell RNA sequencing. Nat Commun 2017, 8 (1): 2128.

[6] Costa V, Angelini C, De FI, et a1. Uncovering me compplexity of transcriptomes witIl RNA-Seq [J]. J Biomed Biotechn01. 2010. 2010: 853916 .

[7] WaIlg Z, Gerstein M, Snyder M. RNA —-Seq: are Volutionary tool for transcriptomics [J] 1. Nat Rev Genet, 2009, 10 (1): $57-63$.

[8] MA SufangYAN Jinghua. Single-cell sequencing technology and its application in infectious disease [J]. Biotechnology \& Business, 2018, (2): 85-90.

[9] Whitesides GM. The origins and the future of microfluidics [J]. Nature, 2006, 442 (7101): 368-373.

[10] LeGac S, Nordhoff V. Microfluidics for mammalian embryo cultureand selection:where do we stand now [J]. Mol Hum Reprod, 2017, 23 (4): 213.

[11] Zhang Q, Wang T, Zhou Q, et al. Development of a facile dropletbased single- cell isolation platform for cultivation and genomic analysis in microorganisms $[\mathrm{J}]$. Scientific Reports, 2017, 7 (1): 21-27.

[12] SONG Zhang-yongWANG Guang-xi. Progress in single cell sequencing to study pathogenic microbes in humans [J]. Journal of Parasitic Biology, 2017, 12 (11): 1116-1118.

[13] Navin NE. Cancer genomics: one cell at a time [J]. Genome Biol, 2014, 15:452.

[14] Darrell L, Ellsworth, Heather L, et al. Single- cell sequencing and tumorigenesis: improved understanding of tumor evolution and metastasis [J]. Clin Trans Med, 2017, 6 (1): 15.

[15] Grun D. Design and ananlysis of a single-cell sequencing experiments [J]. Cell, 2015, 163 (4): 799-810.

[16] Chen C, Xing D, Tan L, et al. Single-cell whole-genome analyses by Linear Amplification via Transposon Insertion (LIANTI) [J]. Science, 2017, 356 (6334): 189-194.

[17] Hedlund E, Deng Q, et al. Single-cell RNA sequencing: Technical advancements and biological applications [J]. Molecular Aspects of Medicine, 2017, 1: 1-11.

[18] Tang F, Barbacioru C. mRNA-Seq whole-transcriptome analysis of a single cell [J]. Nat Methods, 2009, 6 (5): 377-382.

[19] Sasagawa Y, Nikaido I, Hayashi T, et al. Quartz-Seq: a highly reproducible and sensitive single-cell RNA sequencing method, reveals non-genetic gene-expression heterogeneity $[\mathrm{J}]$. Genome Biol, 2013, 14 (4): 31.

[20] Datlinger P, Rendeiro AF, Schmidl C, et al. Pooled CRISPR screening with single-cell transcriptome readout $[\mathrm{J}]$. Nature Methods, 14 (3): 297-301.

[21] Habib N, Avraham-Davidi I, Basu A, et al. Massively parallel single-nucleus RNA-seq with DroNc-seq [J]. Nature Methods, 14(10): 955-958.

[22] Adkar, S. S. et al. Genome engineering for personalized arthritis therapeutics. Trends Mol. Med. 23, 917-931 (2017).

[23] Nam, Y., Rim, Y. A., Jung, S. M. \& Ju, J. H. Cord blood cell-derived iPSCs as a new candidate for chondrogenic differentiation and cartilage regeneration. Stem Cell Res. Ther. 8,16 (2017).

[24] Koyama, N. et al. Human induced pluripotent stem cells differentiated into chondrogenic lineage via generation of mesenchymal progenitor cells. Stem Cells Dev. 22, 102-113 (2012).

[25] Craft, A. M. et al. Generation of articular chondrocytes from human pluripotent stem cells. Nat. Biotechnol. 33, 638 (2015).

[26] Yamashita, A. et al. Generation of scaffoldless hyaline cartilaginous tissue from human iPSCs. Stem Cell Rep. 4, 404-418 (2015).

[27] Chan, C. K. et al. Identification of the human skeletal stem cell. Cell 175, 43-56. e21 (2018).

[28] Loh, K. M. et al. Mapping the pairwise choices leading from pluripotency to human bone, heart, and other mesoderm cell types. Cell 166, 451-467 (2016).

[29] Adkar, S. S. et al. Step-wise chondrogenesis of human induced pluripotent stem cells and purification via a reporter allele generated by CRISPR-Cas9 genome editing. Stem Cells 37, 65-76 (2019). 
[30] Wu CL, Dicks A, Steward N, Tang R, Katz DB, Choi YR, Guilak F. Single cell transcriptomic analysis of human pluripotent stem cell chondrogenesis. Nat Commun. 2021 Jan 13; 12 (1): 362. doi: 10.1038/s41467-020-20598-y. PMID: 33441552; PMCID: PMC7806634.

[31] Kempf, H. et al. Bulk cell density and Wnt/TGFbeta signalling regulate mesendodermal patterning of human pluripotent stem cells. Nat. Commun. 7, 13602 (2016).

[32] Sun H, Wen X, Li H, et al. Single-cell RNA-seq analysis identifies meniscus progenitors and reveals the progression of meniscus degeneration [J]. Annals of the Rheumatic Diseases, 2019, 79 (3): annrheumdis-2019-215926.

[33] Baryawno N, Przybylski D, Kowalczyk MS, et al. A cellular taxonomy of the bone marrow stroma in homeostasis and leukemia. Cell 2019; 177: 1915-32.

[34] Ji Q, Zheng Y, Zhang G, et al. Single-cell RNA-seq analysis reveals the progression of human osteoarthritis. Ann Rheum Dis 2019; 78: 100-10.

[35] Dunn SL, Soul J, Anand S, et al. Gene expression changes in damaged osteoarthritic cartilage identify a signature of non-chondrogenic and mechanical responses. Osteoarthritis Cartilage 2016; 24: 1431-40.

[36] Gunja NJ, Athanasiou KA. Passage and reversal effects on gene expression of bovine meniscal fibrochondrocytes. Arthritis Res Ther 2007; 9.

[37] Baker J, Falconer AMD, Wilkinson DJ, et al. Protein kinase D3 modulates MMP1 and MMP13 expression in human chondrocytes. PLoS One 2018; 13: e0195864.

[38] Won Y, Shin Y, Chun C-H, et al. Pleiotropic roles of metallothioneins as regulators of chondrocyte apoptosis and catabolic and anabolic pathways during osteoarthritis pathogenesis. Ann Rheum Dis 2016; 75: 2045-52.

[39] Puram SV, Tirosh I, Parikh AS, et al. Single-cell transcriptomic analysis of primary and metastatic tumor ecosystems in head and neck cancer. Cell 2017; 171: 1611-24.

[40] Berent-Maoz B, Montecino-R odriguez E, Signer RAJ, et al. Fibroblast growth factor-7 partially reverses murine thymocyte progenitor aging by repression of INK4A. Blood 2012; 119: 5715-21.

[41] Abhishek A, Doherty M. Diagnosis and clinical presentation of osteoarthritis. Rheum Dis Clin North Am. 2013; 39 (1): 45-66.

[42] Hawker GA. Osteoarthritis is a serious disease. Clin Exp Rheumatol. 2019; 37 (Suppl 120): 3-6.
[43] Li X, Liao Z, Deng Z, Chen N, Zhao L. Combining bulk and single-cell RNA-sequencing data to reveal gene expression pattern of chondrocytes in the osteoarthritic knee. Bioengineered. 2021 Dec; 12 (1): 997-1007. doi: 10.1080/21655979.2021.1903207. PMID: 33749514.

[44] Fisch KM, Gamini R, Alvarez-Garcia O, et al. Identification of transcription factors responsible for dysregulated networks in human osteoarthritis cartilage by global gene expression analysis. Osteoarthritis Cartilage. 2018; 26 (11): 1531-1538.

[45] Ajekigbe B, Cheung $\mathrm{K}, \mathrm{Xu} \mathrm{Y}$, et al. Identification of long non-coding RNAs expressed in knee and hip osteoarthritic cartilage. Osteoarthritis Cartilage. 2019; 27 (4): 694-702.

[46] Chen H, Chen L. An integrated analysis of the competing endogenous RNA network and co-expression network revealed seven hub long non-coding RNAs in osteoarthritis. Bone Joint Res. 2020; 9 (3): 90-98.

[47] Karlsson C, Dehne T, Lindahl A, et al. Genome-wide expression profiling reveals new candidate genes associated with osteoarthritis. Osteoarthritis Cartilage. 2010; 18 (4): 581-592.

[48] Soul J, Dunn SL, Anand S, et al. Stratification of knee osteoarthritis: two major patient subgroups identified by genome-wide expression analysis of articular cartilage. Ann Rheum Dis. 2018; 77 (3): 423.

[49] Quanbo J, Yuxuan Z, Guoqiang Z, et al. Single-cell RNA-seq analysis reveals the progression of human osteoarthritis [J]. Annals of the Rheumatic Diseases, 2018: annrheumdis-2017-212863.

[50] Fernandes L M, Khan N M, Trochez C M, et al. Single-cell RNA-seq identifies unique transcriptional landscapes of human nucleus pulposus and annulus fibrosus cells [J]. Scientific Reports, 2020, 10 (1): 15263.

[51] Rodrigues-Pinto, R., Richardson, S. M. \& Hoyland, J. A. An understanding of intervertebral disc development, maturation and cell phenotype provides clues to direct cell-based tissue regeneration therapies for disc degeneration. Eur. Spine J. 23, 1803-1814.

[52] Aszódi, A., Chan, D., Hunziker, E., Bateman, J. F. \& Fässler, R. Collagen II is essential for the removal of the notochord and the formation of intervertebral discs. J. Cell Biol. 143, 1399-1412. https://doi.org/10.1083/jcb.143.5.1399 (1998).

[53] ZHANG Yu. Application of single cell sequencing technology in bone and joint diseases [J]. Orthopedic Journal of China, v. 28; No. 485 (03): 235-238. 